\title{
The clinical effects of CPR meter on chest compression quality: a QI project.
}

\section{Christopher Picard, Richard Drew, Domhnall O'Dochartaigh, Matthew J Douma, Candice Keddie, Colleen Norris.}

Background: High-quality chest compressions are the cornerstone of resuscitation. Training guidelines require CPR feedback, and pre-clinical data shows that feedback devices improve chest compression quality; but devices are not being used in many emergency departments, and their impact on clinical care is less well understood. Some services use defibrillator generated reports for quality improvement, but these measurements may be limited in scope and have not been rigorously compared to other tools.

Methods: Laerdal CPRMeter 2 chest compression feedback devices were purchased using funds made available by a zone QI initiative. Initial training for implementation consisted of staff performing one minute of blinded chest compression using the feedback device, followed by one minute of chest compression unblinded. Staff were shown the raw percentage of chest compressions meeting target depth, release, and rate under both conditions as well as overall improvement. Following initial orientation, devices were incorporated into clinical care and all subsequent staff simulation and training. Clinically, use of the feedback device and completion or QI tracking forms was not mandated but was encouraged by drawing code participant names from completed forms for a free ACLS or PALS course. Data from all codes were automatically collected by the LifePak 20, data from any resuscitation using the Laerdal CPRmeter 2 were also automatically recorded when the device was used: these data were downloaded weekly. Completed questionnaire forms were submitted to the Clinical Educators and extracted as received.

Evaluation Methods: Chest compression quality data was collected in two ways: first, using a Laerdal CPRMeter2, second, by downloading and analyzing cardiac arrest data from a LifePak20 defibrillator using CodeStatTM software. Device data were matched and synthesized by an emergency department CNE using Microsoft excel and IBM SPSS 26. Descriptive statistics (mean and standard deviations) are used to describe the data. Differences in chest compression quality and duration of resuscitations between resuscitation that did or did not use a feedback device or a backboard were compared using independent t-testing. Differences in chest compressions at the target depth, release, and rate between the numbers of staff involved were assessed using ANOVA. Agreement between devices (CPRMeter2 and LifePak) used during the resuscitations were evaluated using paired t-testing, Pearson correlations, and Bland-Altman plots. All tests were two-tailed with predetermined significance levels set at $\mathrm{a}=0.05$.

Results: Data collection occurred between August 2019 and December 2020. There were a total of 50 cardiac arrests included, 36 had questionnaire data returned, 36 had data collected from the CPR meter 2, 24 had data collected from the LifePak, and 10 had data collected using all three methods. The average duration of resuscitation (number of chest compressions) was 1079.56 $(\mathrm{SD}=858.25$ ); there was no difference in the duration of resuscitation (number of chest 
compressions) between resuscitations using versus not using CPR feedback devices $(p=0.673)$. Resuscitations utilizing chest compression feedback had a higher percentage of chest compressions at the target rate compared to resuscitations not using feedback $(74.08 \% \mathrm{vs}$ $42.18 \%, \mathrm{p}=0.007)$. Resuscitations that utilized a backboard had a higher percentage of chest compressions at target depth $(72.92 \%$ vs $48.73 \%, \mathrm{p}=0.048)$. There were no differences noted in the duration of resuscitation attempt $(\mathrm{p}=0.167)$ or percentages of chest compressions at the target depth $(\mathrm{p}=0.181)$, release $(\mathrm{p}=0.538)$, or rate $(\mathrm{p}=0.656)$ between resuscitations with different sized teams $(4-5,6-7,8-9,>10$ staff involved). There was a strong positive correlation $(\mathrm{r}=0.771$, $\mathrm{p}=0.005, \mathrm{n}=11$ ) between the two measurement methods and chest compression rates, and no statistically significant difference in measured scores $(\mathrm{p}=0.999)$, with $100 \%$ of values falling within the Bland-Altman confidence intervals of 36.72 and $-36.72, n=11$. Interpretation of the levels of agreement between these two device measures methods should be done cautiously however, given the small sample size and wide confidence intervals.

\section{Implications}

1) Incorporation of visual chest compression feedback and use of a backboard are fast and affordable and significantly improved the percentage of chest compression at the target rate and depth.

2) There was no correlation between the size of the resuscitation team and the percentage of chest compressions at the target depth, release or rate; nor was the feedback device use associated with the duration of the resuscitation attempt.

3) The implications of improvement with the CPR meter suggests that areas or service not using feedback should consider implementing its use to achieve the target compression rate.

4) Compared to LifePak feedback alone the CPRMeter2 will also allow services to target depth and release targets as well as rate. 